\title{
Evolución del sistema de ciudades en la CEE (1970-1986). Repercusiones tras la entrada de Grecia, España y Portugal
}

\author{
Vicente Ortells Chabrera *
}

\section{INTRODUCCION}

El modelo de población urbana/rural con límites definidos, ha cambiado sustancialmente en las dos últimas décadas. Los espacios periurbanos enlazan el campo con la ciudad y muchas residencias secundarias se han reconvertido en "urbanizaciones dormitorio". Europa occidental ejemplifica este fenómeno, que se nutre de la pérdida de efectivos en las grandes ciudades.

Se estudia seguidamente la red europea de ciudades medias y grandes con censo mínimo de 100.000 habitantes, analizando la evolución experimentada desde 1970 hasta 1986. La inclusión de los paises del sur de Europa (Grecia, España, Portugal) en la CEE, con demografía más dinámica, ha consolidado la red urbana mediterránea, equilibrando a su vez el espacio urbano europeo.

(*) Centro Asociado UNED. Vila-real. 


\section{PROBLEMATICA DE LAS FUENTES: UNIFICACION NECESARIA}

Las principales fuentes utilizadas son los Anuarios Demográficos de Naciones Unidas, que registran los censos de las distintas naciones. El principal problema es la variación en los registros de población. También son notables las ausencias de datos o las cifras un tanto anómalas, aunque en nuestro caso son complementarias y no hacen variar los resultados del análisis. Para completar las tablas se han utilizado distintas ediciones del Calendario de Agostini y del Anuario El Pais de 1987. Pese a todo, consideramos que el análisis comparativo es válido y las conclusiones se ajustan a la realidad.

Por otra parte, hay que reflexionar sobre la necesidad a todos los efectos de unificar criterios estadísticos y fechas de registro en el ámbito de la CEE, medida que también suprimiría fronteras en la investigación y planificación conjunta del espacio comunitario.

\section{EVOLUCION DE LA RED URBANA (1970-1986)}

La población de Europa occidental, tras la recuperación demográfica posbélica, acudió en gran número a las ciudades, que tuvieron su "década prodigiosa" en los sesenta. La urbanización continuó en los años siguientes y se ha ralentizado en la actualidad, con la aparición de nuevos espacios periurbanos, la consolidación de las ciudades pequeñas y la tendencia centrifuga en las macrourbes.

\section{El auge de las ciudades de tipo medio}

El grupo de ciudades con censos entre 100.000 y medio millón de habitantes es el que ha experimentado el mayor incremento en la red urbana de la CEE. En 1970 eran 236, llegando a 261 quince años después y registrando un aumento aritmético de población del 13 por 100. 
En estas cifras desempeñan un papel muy importante los países del sur de Europa, concretamente España y Grecia. De las 25 ciudades que superaron 100.000 habitantes en los ochenta, nada menos que 14 eran españolas y la mitad de ellas situadas en el área de Madrid. Baste recordar el ejemplo de Fuenlabrada (7.369 h. en 1970 y 119.848 en 1986), extraordinario exponente de la descongestión experimentada por las grandes urbes europeas, de las que Madrid es el mejor ejemplo. Así pues, la entrada de España en la CEE ha reforzado considerablemente este segmento urbano que es uno de los que ofrecen por su rango y tamaño las mejores perspectivas de futuro.

Contrariamente a lo sucedido en España, en los paises europeos con mayor tradición urbana se ha producido un receso en beneficio de las ciudades más pequeñas. El Reino Unido es el país que mejor refleja esta orientación hacia un hábitat semiurbano, mientras que Francia y la RFA mantienen una evolución mínima o se ha iniciado el descenso sobre el total de población (Italia, tabla I).

\section{Recesión en las grandes ciudades}

El grupo de ciudades entre 500.000 y 1.000 .000 de habitantes es el que posee un mayor desequilibrio espacial. En 1970, cuatro ciudades se situaban en los midlands ingleses y 11 en el área del Rhin, 15 de un total de 26. Actualmente, dos ciudades inglesas, una francesa y una cuarta holandesa han bajado del medio millón y España sustituye al Reino Unido en el segundo lugar comunitario en cuanto al número de ciudades en este grupo.

Si la evolución global ha sido negativa, con pérdidas del $-12,3$ por 100 de población, en España sucede nuevamente lo contrario y ésta se duplica ampliamente (114 por 100). Sin embargo, en las ciudades millonarias se pierde un $-4,3$ por 100 , exactamente igual a lo sucedido en todos los demás paises del Mercado Común, con un valor global de descenso de $-10,7$ por 100. En definitiva, las macrourbes y ciudades medias europeas han declinado su extraordinario aumento anterior en beneficio de sus áreas metropolitanas y de las ciudades pequeñas, aunque ninguna de ellas ha perdido su rango y papel rector. 


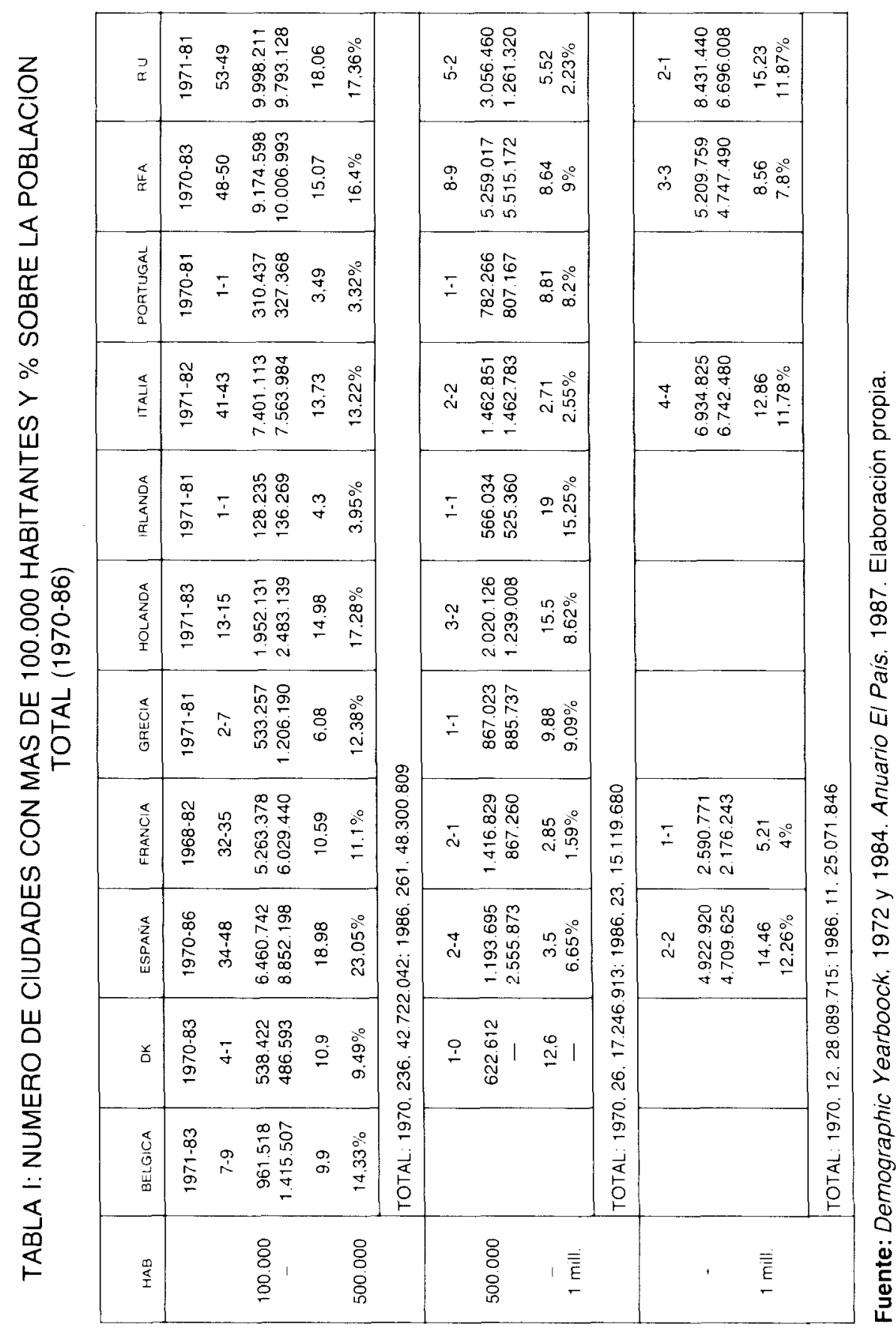


La política de descongestión de las grandes capitales se ha seguido en toda la Europa comunitaria, especialmente en el Reino Unido y la RFA, tratando de evitar el gigantismo urbano y buscando las "metrópolis de equilibrio", es decir, que la futura Europa de las ciudades debería ser un organismo policéntrico (COMPAGNA, 1974, pág. 242), con reparto equitativo de los poderes de decisión.

\section{DISTRIBUCION ESPACIAL DE LA RED URBANA}

Para medir la distribución espacial de las ciudades europeas se ha elaborado un índice deducido de la densidad de población, al que llamamos ÍNDICE DE DENSIDAD ESPACIAL DE NÚCLEOS (ID).

$$
I D=P \times N
$$

$\mathrm{P}=$ población total de las ciudades del país.

$\mathrm{N}=$ número total de ciudades.

$\mathrm{S}=$ superficie de cada Estado $\left(\mathrm{Km}^{2}\right)$.

Los resultados (tabla II) confirman lo que parece intuirse de la observación de los mapas (Figs. 1 y 2) y permiten la división del ámbito comunitario en tres espacios diferenciados.

\section{Altas densidades espaciales en el norte de Europa}

Los paises con mayores índices de densidad espacial son la RFA, Reino Unido, Italia y Holanda. Al urbanismo tradicional que creaba centros macrocéfalos - como sucede en los países en vías de desarrollo- ha sucedido en las áreas de sólido desarrollo la aparición de auténticas regiones urbanas, que 
VICENTE ORTELLS CHABRERA

\begin{tabular}{|c|c|c|c|c|c|c|c|c|}
\hline & 面 & 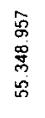 & $\underset{\stackrel{m}{\circ}}{ }$ & $\begin{array}{l}\hat{今} \\
\stackrel{0}{0} \\
0 \\
0 \\
0 \\
0\end{array}$ & ธ్ & 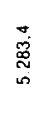 & 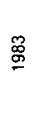 & 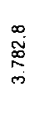 \\
\hline 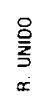 & $\stackrel{\mathscr{0}}{\circ}$ & 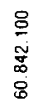 & 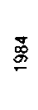 & 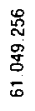 & $\stackrel{\mathscr{0}}{\circ}$ & 薦 & 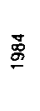 & 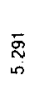 \\
\hline 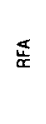 & 寅 & 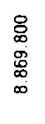 & 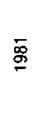 & 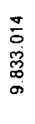 & $\underset{\sigma}{\bar{\sigma}}$ & $\stackrel{\widetilde{\sim}}{\sim}$ & 畺 & $\stackrel{\varphi}{\sim}$ \\
\hline 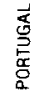 & 尽 & $\begin{array}{l}\text { 总 } \\
\text { 总 }\end{array}$ & $\stackrel{\bar{g}}{\stackrel{9}{2}}$ & $\begin{array}{l}\stackrel{8}{0} \\
\text { id } \\
\text { d. }\end{array}$ & & & & \\
\hline$\stackrel{x}{\exists}$ & $\overline{\underline{\sigma}}$ & 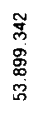 & $\stackrel{\mathscr{I}}{\mathbb{g}}$ & 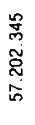 & $\underline{\underline{\sigma}}$ & 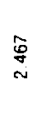 & $\stackrel{\mathscr{ٌ}}{\stackrel{\mathscr{g}}{2}}$ & $\underset{\sim}{\stackrel{\infty}{*}}$ \\
\hline 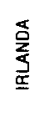 & बิ & 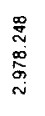 & 嘿 & 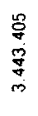 & స్ & $\bar{i}$ & $\stackrel{\text { 兽 }}{ }$ & 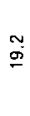 \\
\hline $\begin{array}{l}\text { 웧 } \\
\text { 옫 }\end{array}$ & $\stackrel{2}{\stackrel{9}{\sigma}}$ & $\begin{array}{l}\overline{0} \\
\infty \\
\infty \\
\tilde{D} \\
\underline{0}\end{array}$ & 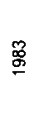 & 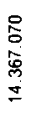 & $\stackrel{\circ}{\stackrel{\circ}{\sigma}}$ & 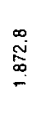 & $\stackrel{\mathbb{g}}{\stackrel{g}{9}}$ & 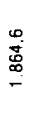 \\
\hline 志 & $\bar{\Phi}$ & $\begin{array}{l}0 \\
\stackrel{0}{0} \\
0 \\
0 \\
\infty \\
\infty\end{array}$ & 嘿 & 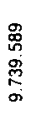 & న్ & $\stackrel{\simeq}{\cong}$ & 嘿 & $\underset{\substack{0 \\
0}}{2}$ \\
\hline $\begin{array}{l}\frac{x}{\mathrm{~J}} \\
\frac{\mathrm{x}}{\mathrm{x}} \\
\mathrm{d}\end{array}$ & $\stackrel{\%}{\%}$ & 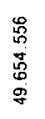 & $\stackrel{8}{0}$ & 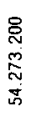 & $\underset{\$}{\$}$ & 号 & 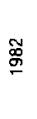 & ثे \\
\hline 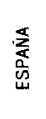 & 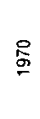 & 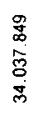 & 喿 & 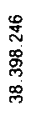 & 号 & $\begin{array}{l}\overrightarrow{0} \\
\stackrel{8}{6}\end{array}$ & 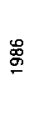 & 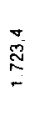 \\
\hline 吕 & $\stackrel{2}{9}$ & 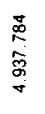 & 离 & 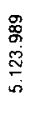 & $\stackrel{?}{\stackrel{2}{\sigma}}$ & 熙 & $\stackrel{\text { 兽 }}{ }$ & $\stackrel{m}{=}$ \\
\hline 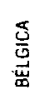 & $\stackrel{乛}{9}$ & $\begin{array}{l}\tilde{N} \\
\tilde{\omega} \\
0 \\
0 \\
0\end{array}$ & 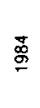 & 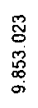 & : & 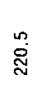 & $\underset{\Phi}{\mathbb{\Phi}}$ & $\begin{array}{l}\dot{\sigma} \\
\tilde{\sigma}\end{array}$ \\
\hline
\end{tabular}


disminuyen la incidencia de las deseconomías de aglomeración nacidas del gigantismo urbano (DERYCKE, 1971, pág. 87). Los midlands ingleses, las cuencas del Ruhr y Rhin, el anillo holandés o el Piamonte y la Lombardía italianos, son claros esponentes de redes densas de ciudades equilibradas. Sin embargo, extensas regiones de estos Estados, como el norte de Gran Bretaña y el sur de Italia, se han vaciado y empobrecido para llenar aquéllas.

La evolución de los ID, ralentizada por la propia recesión demográfica, demuestra una tendencia hacia la estabilización, incluso con cambios significativos a la baja en el Reino Unido $(5.283,4-3.782,8)$. Es deseable que la política regional comunitaria atienda las áreas más deprimidas, que son a su vez las menos urbanizadas, para conseguir un desarrollo armónico en todas sus regiones.

\section{Redes equilibradas en el sur de Europa}

La Europa mediterránea, a excepción de Italia por el elevado papel específico del norte, es el territorio europeo con una red urbana más compensada en su distribución espacial. Los valores de Francia son los más representativos (596-617) y su "armazón urbano" refleja unas relaciones bastante equitativas entre todas sus ciudades (DREYFUS, 1966, pág. 14), exceptuando el peso político/sociocultural de París.

El ID de España se ha doblado desde 1970 a 1986 (946-1.723), como consecuencia del incremento de núcleos con censo superior a 100.000 habiiantes. Sin embargo, la mitad de estas ciudades se sitúan en el cinturón urbano de Madrid acentuándose el desequilibrio entre tres áreas densas (Mediterráneo, Cantábrico y Madrid) y los vacios interiores con islotes urbanos como Zaragoza y Valladolid. En un nivel superior, cabe considerar homogéneo el reparto en grandes urbes, sin ninguna macrocefalia clara (caso de París o Londres), dado el rango y tamaño similar de las áreas urbanas de Madrid y Barcelona.

\section{Los vacíos periféricos}

La menor densidad de ciudades se obtiene en las regiones más extremas, donde la red urbana es muy difusa y con tendencias muy marcadas de macrocefalia, aunque sea en el cómputo de la aglomeración urbana. Ėste 
es el caso de Portugal, con las aglomeraciones de Oporto y Lisboa que totalizan el 30 por 100 de la población del país; Grecia, 42,5 por 100 entre las áreas de Atenas y Tesalónica, o más acusada aún es la macrocefalia de Copenhague (27 por 100), e incluso Dublín (15 por 100), capital del pais menos urbanizado de la CEE junto a Portugal.

Por otra parte, existen zonas marginales en los propios Estados más urbanizados, con escasas ciudades rectoras: los highlands y NW de Escocia o el sur continental de Italia. Asimismo, en una zona común a varios Estados se encuentra un corredor semivacio de ciudades importantes. Entre los $1.600 \mathrm{Km}$ que separan Madrid y Munich, sólo destacan los núcleos de Zaragoza, Toulousse y Lyon, con un hinterland medio de $200 \mathrm{Km}$, que sólo se suaviza en el centro de Francia. Además, este "pasillo europeo» reúne, junto a la periferia comunitaria, las regiones de menor renta per capita (ABEJON y otros, 1986, pág. 38 , mapa).

\section{CONCLUSIONES: EL MAPA EUROPEO DE LAS CIUDADES}

En cuanto a la evolución experimentada por las ciudades europeas desde 1970, se concluyen dos hechos fundamentales:

- Descongestión de las grandes ciudades y aparición de espacios periurbanos alternativos en la línea de las new towns británicas (WYNN, SMITH, 1978).

- Aumento del número de ciudades de tamaño medio, de manera muy notable en el sur de Europa (España, Grecia).

Desde otro punto de vista, el mapa actual de las ciudades en la CEE arroja unos desequilibrios regionales bastante acusados:

- Franja septentrional semivacía, con tan sólo 13 ciudades $(4,4$ por 100 del total) al norte del paralelo 55 (Escocia, Dinamarca).

- Sector central atlántico, con una red excesivamente densa que reúne aproximadamente el 60 por 100 de las ciudades. 
- Pasillo central de baja densidad, con islotes urbanos (Madrid, Zaragoza, Toulousse, Lyon, Munich).

- Distribución armónica en el arco mediterráneo, con equilibrio entre sus macrourbes.

Esta situación, tal vez deba mejorarse por la propia política comunitaria en vistas a reforzar la red de núcleos inferiores a 100.000 habitantes y la del segmento 100.000-500.000. También se concluye que en el espacio de la CEE se tienen que eliminar las macrocefalias y repartir los centros de decisión. De entrada, se sigue este camino con las funciones otorgadas a Bruselas y Estrasburgo.

Finalmente, la inclusión de España en el Mercado Común, ha introducido nuevos planteamientos de conexión con la red urbana europea. Seria positiva la conexión con Portugal tratando de consolidar el sistema urbano por Andalucía (Huelva) y Galicia (Vigo), más viable a corto plazo que la recuperación del oeste (Salamanca, Badajoz). La red del Atlántico francés es débil y podría auparse conectada con la vascocantábrica española. EI eje mediterráneo español (Alicante, Valencia, Barcelona) debe proyectarse por las ciudades del mediodia francés. Finalmente, hay dos ciudades con situación simétrica respecto a los Pirineos, Zaragoza y Toulousse, aunque la frontera natural parece, por ahora, excesiva. 


\section{BIBLIOGRAFIA}

Abejón, M.; Arroyo, F.; Camarero, C. y Morán, J. M. (1986): La Europa de los Doce. Temas Clave n. ${ }^{\circ} 103$, Aula Abierta Salvat, Barcelona, $128 \mathrm{pp}$.

Compagna, F. (1974): La política de la ciudad. Instituto de Estudios de Administración Local, Madrid, 285 pp.

DERYCKE, P-H. (1971): La economía urbana. Instituto de Estudios de Administración Local, Madrid, 271 pp.

DREYFUS, J. (1966): “Investigación y ordenaciones urbanas». Consommation. París, $n .^{\circ} 1$.

ONu (1973): Demographic Yearbook, 1972. Organización Naciones Unidas, Nueva York, $664 \mathrm{pp}$.

ONu (1986): Demographic Yearbook, 1984. Organización Naciones Unidas, Nueva York, $1.150 \mathrm{pp}$.

VV.AA. (1980): Calendario Atlante de Agostini 1981. Ist. Gfco. Agostini, Novara, 784, pp. mapas.

VV.AA. (1987): Anuario El Pais, 1987. Ediciones El País, Madrid, 480 $\mathrm{pp}$.

VV.AA. (1987): Calendario Atlante de Agostini 1987. Instituto Geográfico de Agostini, Novara, 784 pp., mapas.

WYNN, M.; SMITH, R. (1978): “Consideraciones sobre algunos aspectos de las nuevas ciudades británicas". Ciudad y Territorio. Instituto de Estudios de Administración Local, n. ${ }^{\circ}$ 4/78, pp. 101108. 


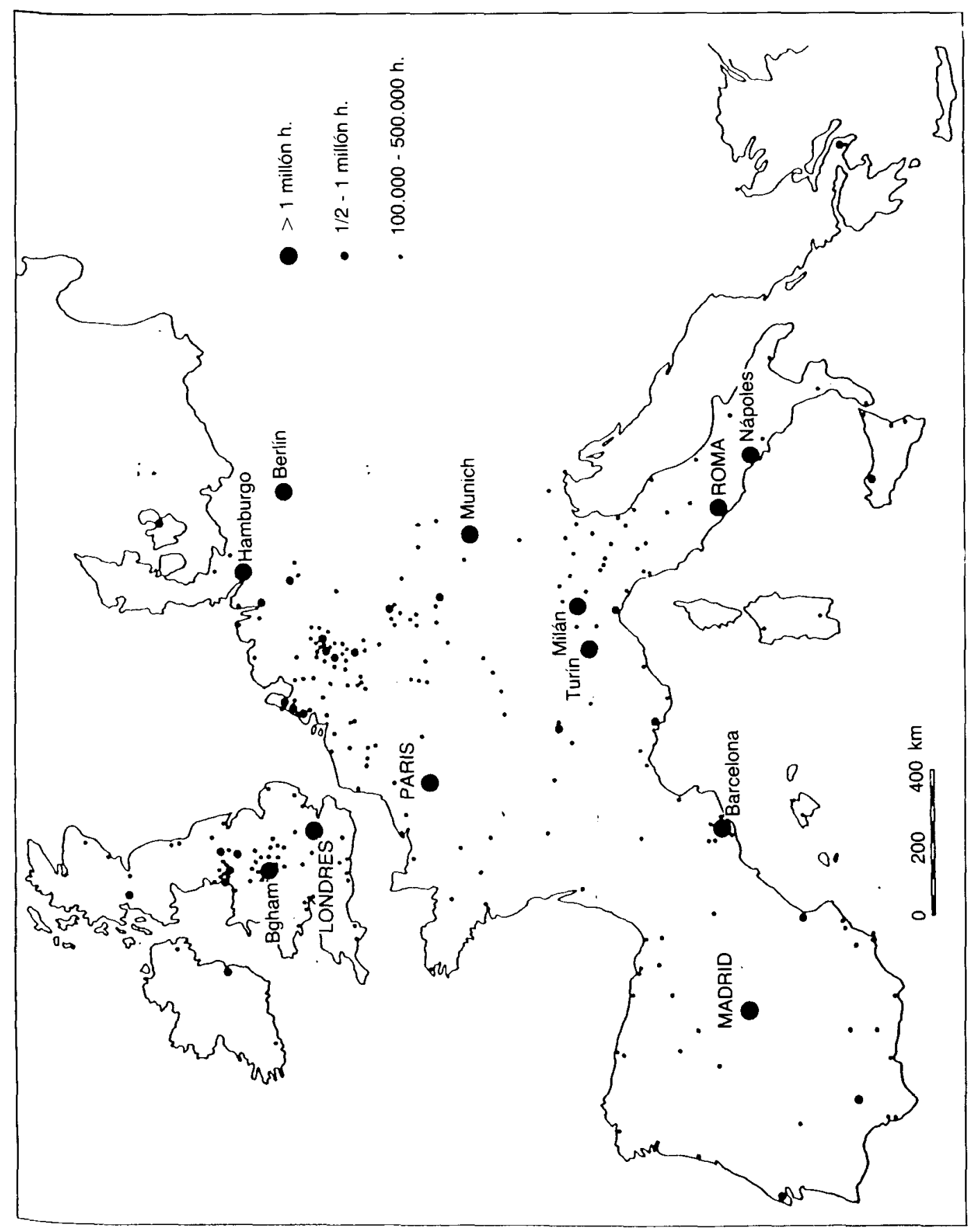




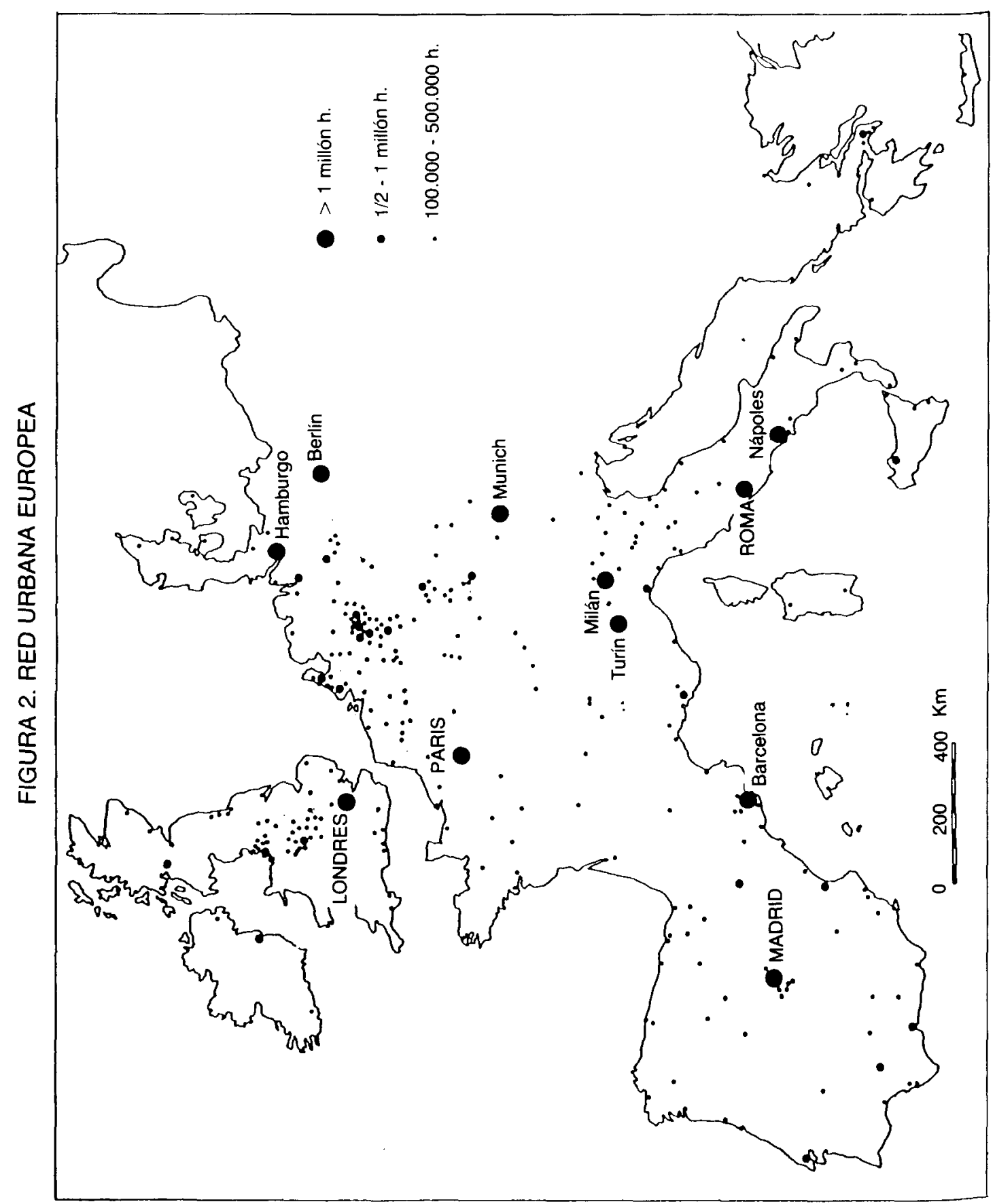




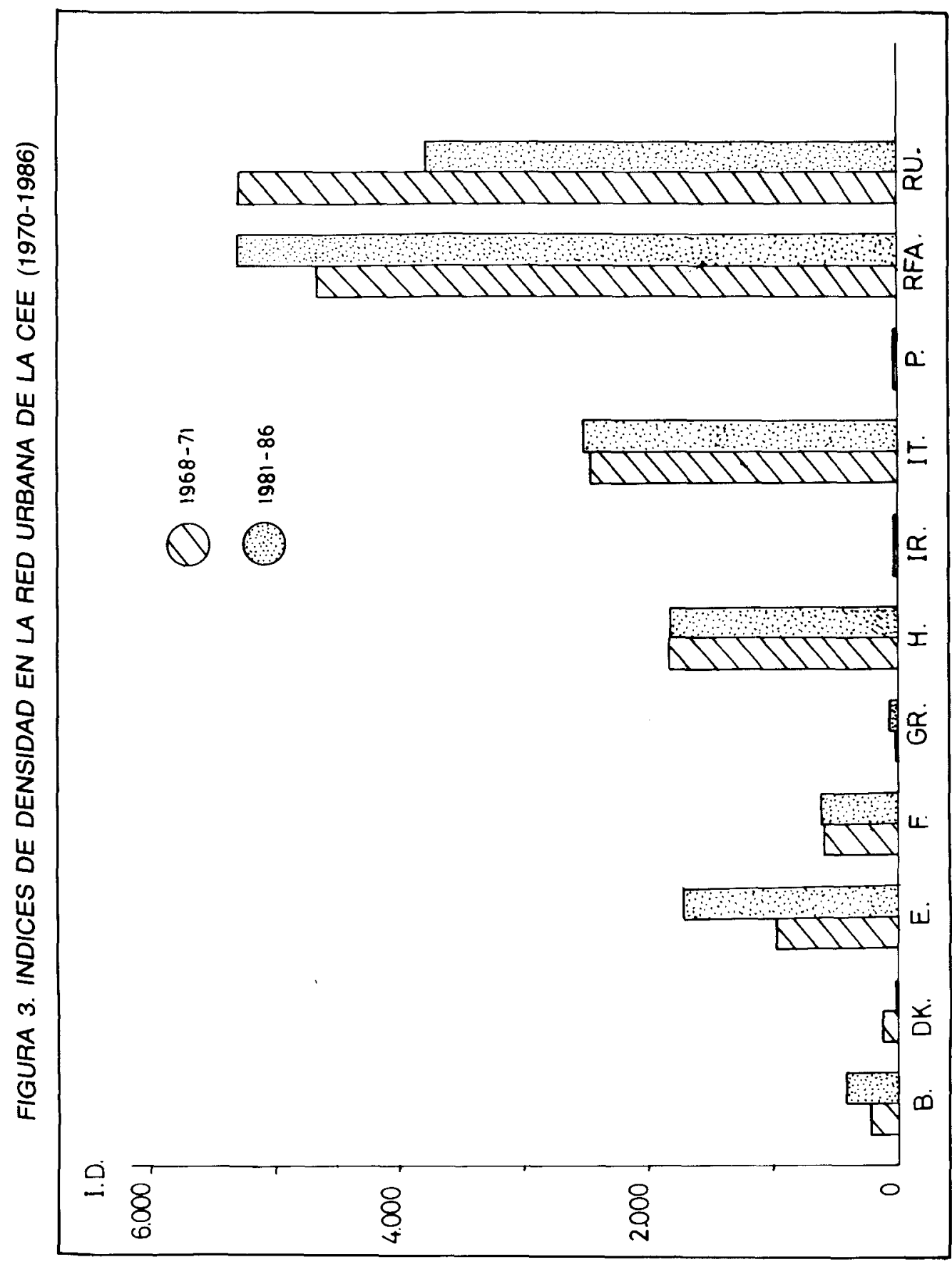

